\title{
Efficient and Reliable Execution of Legacy Codes Exposed as Services
}

\author{
Bartosz Baliś ${ }^{1}$, Marian Bubak $^{1,2}$, Kamil Sterna ${ }^{1}$, and Adam Bemben ${ }^{1}$ \\ ${ }^{1}$ Institute of Computer Science, AGH, al. Mickiewicza 30, 30-059 Kraków, Poland \\ 2 Academic Computer Centre - CYFRONET, Nawojki 11, 30-950 Kraków, Poland \\ \{bubak, balis\}@agh.edu.pl \\ Phone: (+48 12) 61739 64; Fax: (+48 12) 6338054
}

\begin{abstract}
In this paper, we propose a framework that enables fault tolerance and dynamic load balancing for legacy codes running as backends of services. The framework architecture is divided into two layers. The upper layer contains the service interfaces and additional management services, while the legacy backends run in the lower layer. The management layer can record the invocation history or save state of a legacy worker job that runs in the lower layer. Based on this, computing can be migrated to one of a pool of legacy worker jobs. Fault-tolerance in the upper layer is also handled by means of active replication. We argue that the combination of these two methods provides a comprehensive support for efficient and reliable execution of legacy codes. After presenting the architecture and basic scenarios for fault tolerance and load balancing, we conclude with performance evaluation of our framework.
\end{abstract}

Keywords: Legacy code, fault tolerance, load balancing, migration.

\section{Introduction}

Recently developed systems for conducting e-Science experiments evolve into Service-Oriented Architectures (SOA) that support a model of computation based on composition of services into workflows 4. Legacy codes nevertheless, instead of being rewritten or reengineered, are often adapted to new architectures through exposing them as services or components, and remain the computational core of the application. Static and dynamic load balancing (LB) as well as fault tolerance (FT) are highly desired features of a modern execution environment, necessary to sustain the quality of service, high reliability and efficiency. The workflow model, in which the application logic is separated from the application itself, is for this very fact well-suited for LB and FT support, because the execution progress of a workflow is handled by generic services, the enactment engines. However, the presence of legacy jobs running in the backends of services complicates this support, as the legacy jobs are often not directly modeled in the workflow and thus not handled by the same generic enactment engines. While the subjects of FT and LB of parallel and distributed systems [2] are very well recognized for web services [10] [6] or components [8], similar problems for legacy codes running in service backends are still not well addressed. 
This paper presents a solution to support FT and LB for legacy codes exposed as services. We propose a generic framework which enables seamless and transparent checkpointing, migration, and dynamic load balancing of legacy jobs running as backends of services. The proposed framework is based on our previous work, a system for virtualization of legacy codes as grid services, LGF (Legacy to Grid Framework) [1] 11. Unlike our previous work which focused solely on exposing of legacy codes as services, this paper focuses on the aspects of reliable and efficient execution of legacy systems. We propose an architecture for the framework and justify our design choices. We present a prototype implementation of the framework and perform a feasibility study in order to verify whether our solution fulfills functional (FT and LB scenarios) and non-functional (performance overhead evaluation) requirements.

The remainder of this paper is as follows. Section 2 presents related work. Sections 3 and 4 describe the architecture and operation of our FT-LB framework, respectively. We conclude the paper in Section 5 which studies the impact of FT and LB mechanisms on the overall application performance.

\section{State of the Art}

Most existing approaches to adapting legacy codes to modern environments focus on command-line applications and follow a simple adapter pattern in which a web service or a component (e.g. CORBA 5]) wrapper is automatically generated for a legacy command line application, based on a specification of its input parameters 9]. Those approaches differ in terms of addressing other aspects of legacy system's adaptation such as security [7, automatic deployment or integration with a framework for workflow composition. In some cases even some brokering mechanisms are taken into account [3].

Of the available tools, relatively most comprehensive solution is presented by a tool CAWOM [12, wherein one can actually specify the format of legacy system's responses (using a formal language) which allows for more complex interactions with a legacy system, including synchronous and asynchronous calls.

Overall the mentioned tools, whether they offer simple wrapping, or more advanced frameworks with brokering, automatic deployment and workflow composition capabilities, neither take into account nor are designed to support fault tolerance and dynamic load balancing of legacy systems.

Our framework is designed to fill this gap. The separation into two layers, and operation of legacy jobs in a client instead of server fashion, solves many issues and enables flexible solutions of LB and FT problems. We describe those in the following sections of this paper.

\section{LB-FT Framework Architecture}

The architecture of our framework, presented in Fig. 1, is comprised of three main components, namely: Service Client, Service Manager which exposes interfaces, and Backend System on which the legacy code is actually executed. 


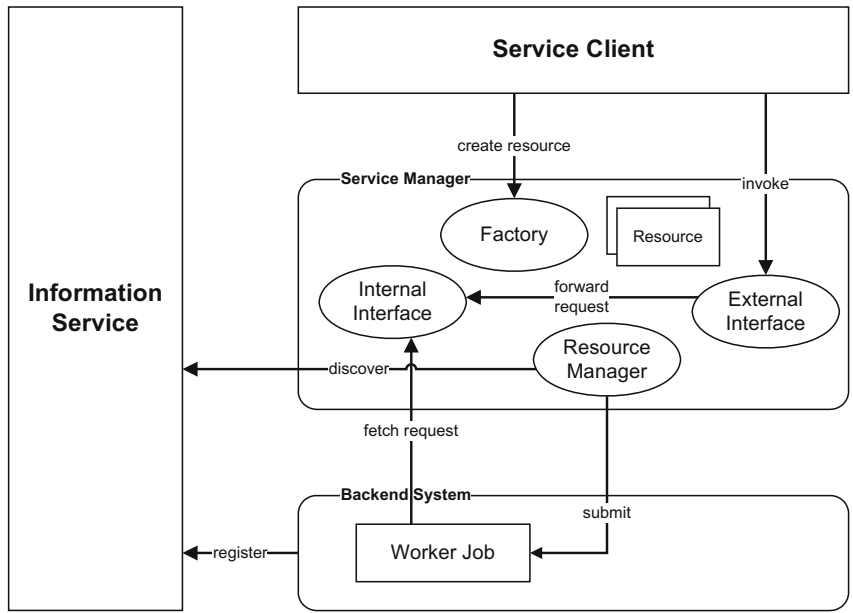

Fig. 1. Architecture of FT \& LB Framework for Legacy Code

The heart of the system is a Service Manager which exposes the legacy code as a service (External Interface) to be invoked by a Service Client. The central concept that enables transparent migration of computation, being a prerequisite for FT and LB, is decoupling of service interface layer from actual legacy code, the latter being deployed as a job (Worker Job), on a (usually remote) machine (Backend System). An important design detail is that the legacy Worker Job acts as a client to the Service Manager and fetches Service Client requests from Internal Interface in the Service Manager. An alternative option would be to use notifications. However, in such a case Worker Jobs would have to act as servers which would make their deployment and migration much more difficult. The remaining component in the Service Manager is a Resource Manager whose main responsibility is to submit Worker Jobs to Backend Systems. In addition, we support stateful conversation with legacy software through WSRF-like services. To this end, a client can create a Resource using a Factory service. In this way, the framework enables stateful, session-based interaction with legacy services.

In the architecture, an external Information System is presented to which Backend Systems register while the Resource Manager acts as a resource broker deciding which Backend System to submit a new legacy Worker Job to, based on a list of available Backend Systems and corresponding monitoring information (such as current load). Alternatively, the Information Service could be replaced by an external Resource Broker to which all resource brokerage decisions would be delegated by the Resource Manager.

Thanks to decoupling of service interfaces and legacy back ends, multiple legacy worker jobs can be connected to a single service as a resource pool. The computation can be easily migrated from one back end to another in case of performance drop (load balancing) or failure (fault tolerance). The framework supports both history-based and checkpoint-based migration of stateful jobs. For 
the former case, the Service Manager records the invocation history of a given client and in case of migration to another worker job, the invocations can be repeated. The latter case is supported by allowing a legacy job to periodically send its state snapshot to the Service Manager; in the event of migration, the new worker job can restore the last state. The architecture enables both lowlevel and high-level checkpointing to create state snapshots, though the current implementation supports only the high-level one in which the worker jobs have to provide an implementation of code to create and restore snapshots.

In our framework, the service interface layer is thin and performs no processing, merely forwarding requests plus other management functions related to migration, state restoration, etc. However, this layer is also subject to failure, e.g. due to software aging of the underlying application containers. Consequently, we also take into account the fault-tolerance of this layer using the technique of active replication. Multiple Service Managers can be assigned to a single interaction between a client and a legacy back end. The client submits all requests to all Service Managers. Similarly, the backend worker job fetches requests from all Service Managers. In consequence, requests are received by the worker job multiple times, however, they are executed only once.

\section{Fault Tolerance and Load Balancing Scenarios}

Fault tolerance and load balancing scenarios for the backend side differ only in the way the migration is initiated. Both scenarios are shown in Fig. 2 (a) and (b) respectively.

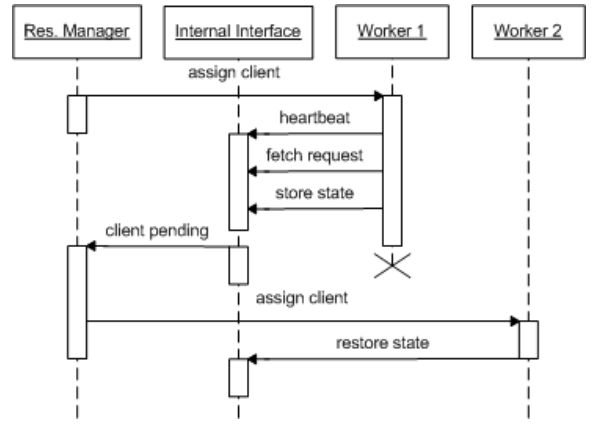

(a)

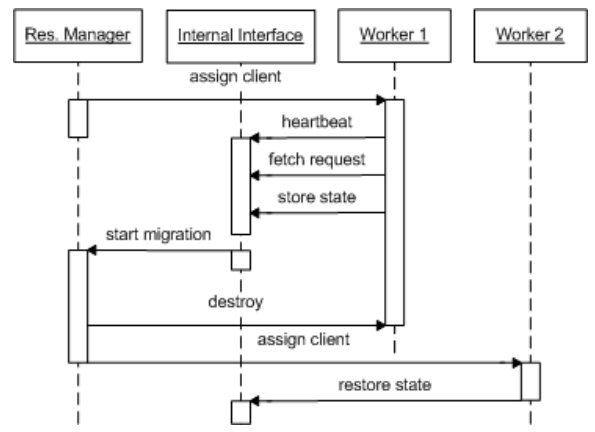

(b)

Fig. 2. Scenarios involving migration: (a) fault tolerance, (b) load balancing

The resource manager fetches a client request (not shown) and assigns a proper worker (assign client). The worker periodically signals the availability (heartbeat), retrieves the client request (fetch request) and stores checkpoints (store state). A migration is triggered either by a worker crash (fault tolerance) or a persistant node overload (load balancing). In the latter case, the worker is 
explicitly destroyed (destroy). In either case, the Service Manager assigns another worker (assign client), which restores the latest state (restore state) and the operation is carried on as it was.

For fault tolerance and software rejuvenation purposes, we employ the active replication mechanism at the upper layer. It is based on multiplying every operation on two (or more) service managers. An appropriate scheme is depicted in Fig. 3. The client calls two service managers at once $(1,2)$. Similarly, the backend worker job fetches requests from both service managers $(3,5)$, however, only one request is actually executed (4). The result is returned to both service managers $(6,7)$ and they forward it back to the client $(8,9)$. When one of service manager crashes (10), the operation is carried on with one service manager only (11-13).

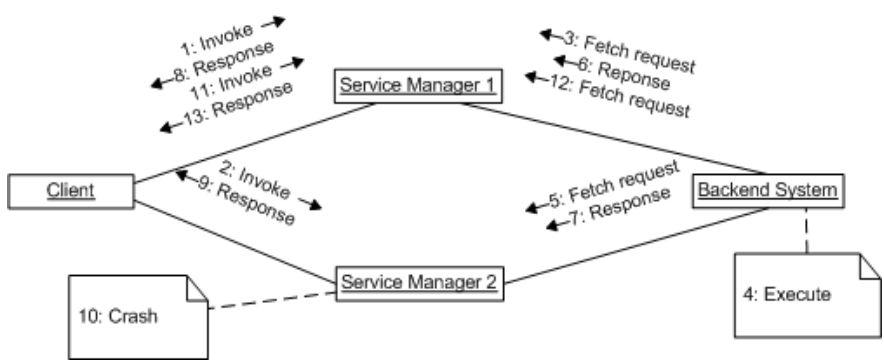

Fig. 3. Sequence of system operations for active replication model

\section{Performance Evaluation}

This section presents an evaluation of the impact of the framework on the overall application's performance. The framework prototype was developed in Java and it generates WSRF services based on Globus Toolkit 4. The following tests were conducted: (1) the impact of the interposition management layer on latency and throughput of a service as compared to direct invocation, (2) the latency of migration in a fault tolerance and load balancing scenario, and (3) the cost of active replication in terms of application's completion time.

For the evaluation, we used a simple algorithm computing a requested number in the Fibonacci sequence, exposed as a service. In total, four IA-32 machines with 1 GB RAM and $1.2 \mathrm{GHz}$ CPU, running Linux Debian, Java 1.4 and Globus 3.2.1 were used.

Fig. 4 (a) shows the overhead of the interposition layer. We compared the performance of two functionally equivalent web services. One of them used a legacy library while the other was standalone. Both web services exposed a single method that calculated the length of a byte array passed as a parameter. Latency and bandwidth of both services were obtained based on the formula:

$$
\text { time }(\text { length })=\text { length/bandwith }+ \text { latency }
$$


Using least squares linear regression fitting, we have obtained figures for both services. As a result, we observed that while latency was increased 2.4 times, bandwidth was reduced only by $12 \%$.

In the fault tolerance scenario, the Service Manager loses connection with one of the workers (at the moment of starting the service on a backend system). After the timeout, the backend system is considered to have undergone abnormal termination. Process migration is initiated, and the method invocations are

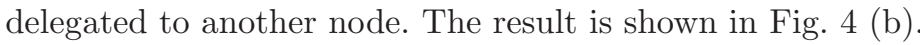

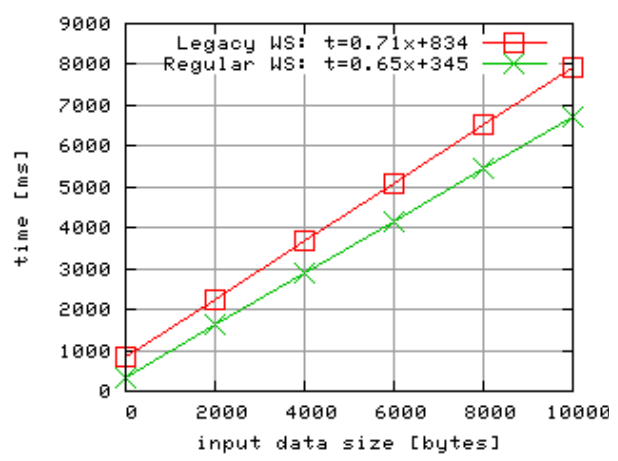

(a)

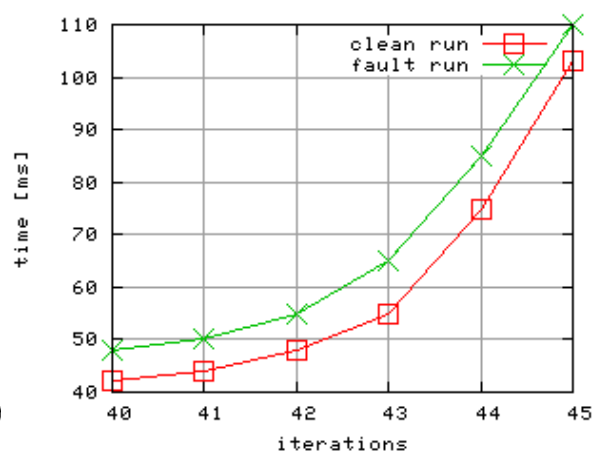

(b)

Fig. 4. (a) Interposition layer overhead (b) Migration overhead

We used history-based fault tolerance whose cost can be estimated at approximately 1-2 seconds. An additional cost is also connected with the number of lost heartbeats before a node is considered to be undergoing a failure.

The load balancing scenario is different only in terms of the cause which triggers the migration, which in this case is a node overload over a certain number of heartbeats. The Service Manager decides to migrate the resources to a node exposing better performance. The overhead proved to be quite similar and is not shown here separately.

Finally, we study the active replication scenario. The framework runs with two service managers present, running in separate containers and performing identical actions. One Service Manager crashes. The platform continues normal work; however the invocations are handled by one service manager only.

The overhead in this scenario is caused by additional processing: on the client side which performs more invocations and has to filter out redundant results, and the backend side where redundant requests have to be discarded and the results has to be returned to more than one Service Manager. Fig. 5 shows the processing time from a worker perspective, for a single, and for two service managers. The times for those two cases are practically the same which proves that the additional processing time - indeed, only limited to more invocations and discarding redundant operations - does not induce substantial overhead. 


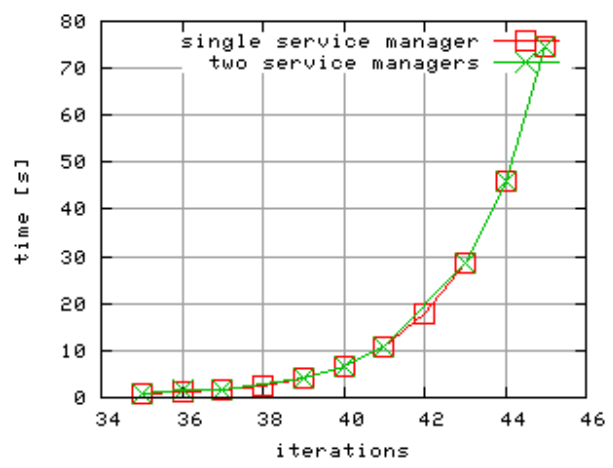

Fig. 5. Active replication overhead

At the same time we observe undisturbed operation of the system despite the crash of one Service Manager.

\section{Conclusion}

We have presented a framework for enabling fault tolerance and dynamic load balancing for legacy codes running in the backend of web services, typically as parts of e-Science workflows. We proposed a two layer architecture in which a management layer containing service interfaces is decoupled from a computational core which is deployed in separate worker jobs.

We use different, complementing strategies for FT \& LB in the two system layers. In the backend layer, we use an efficient method based on a pool of worker jobs of a certain type among which the computing can be switched when there is a need to do so. Recovering state from snapshots or repeating of invocation history can be used in the case of migration of stateful services. In the front-end layer, we use the active replication of service interfaces. Though this method is expensive, it does not require further management such as state snapshots or heartbeat monitoring. Our investigation revealed that thanks to the very architecture of our framework in which the service layer is thin and contains no processing, the inherent overhead of active replication is compensated and is perfectly affordable.

Overall, the performed feasibility study shows the framework fulfills the functional and performance requirements and constitutes a comprehensive solution for reliable and efficient running of workflows that contain legacy code in the computational back ends, which is important for development of e-Science applications.

The future work encompasses, most importantly, the expansion of our system into data-centric workflows involving streaming between legacy jobs, and support for load balancing and fault tolerance scenarios for complex legacy systems, such as parallel MPI applications. Other aspects include integration with a security infrastructure. 
Acknowledgements. This work is partly supported by EU-IST Project CoreGrid IST-2002-004265, and EU-IST Project Virolab IST-027446.

\section{References}

1. Balis, B., Bubak, M., Wegiel, M.: A Solution for Adapting Legacy Code as Web Services. In Proc. Workshop on Component Models and Systems for Grid Applications. 18th Annual ACM International Conference on Supercomputing, Saint-Malo, France, Kluwer (July 2004)

2. Cao, J., Spooner, D. P., Jarvis, S. A., Nudd, G. R.: Grid Load Balancing Using Intelligent Agents. Future Generation Computer Systems special issue on Intelligent Grid Environments: Principles and Applications, 21(1) (2005) 135-149

3. Delaittre, T., Kiss, T., Goyeneche, A., Terstyanszky, G., Winter, S., Kacsuk, P.: GEMLCA: Running Legacy Code Applications as Grid Services. Journal of Grid Computing Vol. 3. No. 1-2. Springer Science + Business Media B.V. (June 2005) 75-90

4. E-Science 2006, Homepage: http://www.escience-meeting.org/eScience2006/

5. Gannod, G. C., Mudiam, S. V., Lindquist, T. E.: An Architecture Based Approach for Synthesizing and Integrating Adapters for Legacy Software. Proc. 7th Working Conference on Reverse Engineering. IEEE (November 2000) 128-137

6. Hwang, S., Kesselman, C.: A Flexible Framework for Fault Tolerance in the Grid. Journal of Grid Computing 1(3)(2003) 251-272

7. Kandaswamy, G., Fang, L., Huang, Y., Shirasuna, S., Marru, S., Gannon, D.: Building Web Services for Scientific Grid Applications. IBM Journal of Research and Development, Vol. 50. No. 2/3 (March/May 2006) 249-260, .

8. Moser, L., Melliar-Smith, P., Narasimhan, P.: A Fault Tolerance Framework for CORBA. International Symposium on Fault Tolerant Computing (Madison, WI) (June 1999) 150-157

9. Pierce, M., Fox, G.: Making Scientific Applications as Web Services. Web Computing (January/Februray 2004)

10. Tartanoglu, F., Issarny, V., Romanovsky, A., Levy, N.: Dependability in the Web Services Architecture. Architecting Dependable Systems. LNCS 2677 (June 2003)

11. Wegiel, M., Bubak, M., Balis, B.: Fine-Grain Interoperability with Legacy Libraries Virtualized as Web Services. Proc. Grid-Enabling Legacy Applications and Supporting End Users Workshop within the framework 15th IEEE HPDC 15, Paris, France ( June 2006)

12. Wohlstadter, E., Jackson, S., Devanbu, P.: Generating Wrappers for Command Line Programs: The cal-aggie wrap-o-matic Project. Proc. 23rd International Conference on Software Engineering (ICSE 2001). ACM (2001) 243-252 